\title{
Envisaging Performance Metrics of ZigBee Wireless Sensors by Topology Variations
}

\author{
Kompal Gupta \\ Electronics Technology \\ Department \\ Guru Nanak Dev University, \\ Amritsar
}

\author{
Rajan Vohra \\ Electronics Technology \\ Department \\ Guru Nanak Dev University, \\ Amritsar
}

\author{
Ravinder Singh Sawhney \\ Electronics Technology \\ Department \\ Guru Nanak Dev University, \\ Amritsar
}

\begin{abstract}
The IEEE 802.15.4 the ZigBee protocol finds numerous applications for commercial buildings and home automation, security, healthcare, medical monitoring, vehicle monitoring, agriculture and environmental monitoring etc. In this paper we have evaluated the effect of topologies variation i.e. Tree, Star and Mesh on load, delay and throughput in different bands using Zigbee wireless sensors by means of OPNET modeler. It is observed that star, tree and mesh topologies have less delay and maximum throughput in $2450 \mathrm{MHz}$ band as compared to other bands i.e. $915 \mathrm{MHz}$ and $868 \mathrm{MHz}$. The obvious reason for this observation can be less resource overheads in $2450 \mathrm{MHz}$ band than other two bands resulting in better network metrics.
\end{abstract}

\section{Keywords}

Delay; Load; Throughput; Topology; ZigBee

\section{INTRODUCTION}

ZigBee standard was developed by IEEE 802.15 .4 [1-4] Task Group and ZigBee Alliance in order to meet the requirements such as low cost, ultra- low power consumption, cheap and easy installation, flexible and extendable networks [5].

ZigBee devices can be either full functional devices (FFD) or reduced functional device (RFD)[6,7]. For example, coordinators and routers are FFD and end devices are RFD. The coordinator provides the initialization, maintenance, and control functions for the network. ZigBee router routes the message within a network. ZigBee has four layers: physical, medium access control, network and application layers. Application layer is responsible for building automation, temperature control, industrial control and wireless sensor monitoring. Network layer is responsible for network management and addressing. Medium Access Control Layer determines the source and destination address of frames and physical Layer is responsible for data transmission service $[8,9]$.

The communication in ZigBee can occur in $868 \mathrm{MHz}$, $915 \mathrm{MHz}$ or $2.4 \mathrm{GHz}$ industrial scientific and medical (ISM) bands [10]. ZigBee devices can use any of these bands for communication; the $2.4 \mathrm{GHz}$ band is more famous as it is open in most of the countries worldwide. The $868 \mathrm{MHz}$ band is specified for European use whereas the $915 \mathrm{MHz}$ band can only be used in the United States, Canada and a few other countries and territories that accept the FCC regulations. To modulate the radio frequency carrier at $2.4 \mathrm{GHz}$, ZigBee uses direct sequence spread spectrum and an offset quadrature phase shift keying (O-QPSK) with half-sine pulse shaping. The applicable area of ZigBee includes home automation [11], consumer electronics, industrial controls and games.

\section{TOPOLOGY}

\subsection{Star Topology}

Figure 1 shows the star topology, the coordinator is at the center and end devices are connected to it. The communication between ZigBee end devices (ZED) is through the ZigBee Coordinator (ZC) [12, 13].

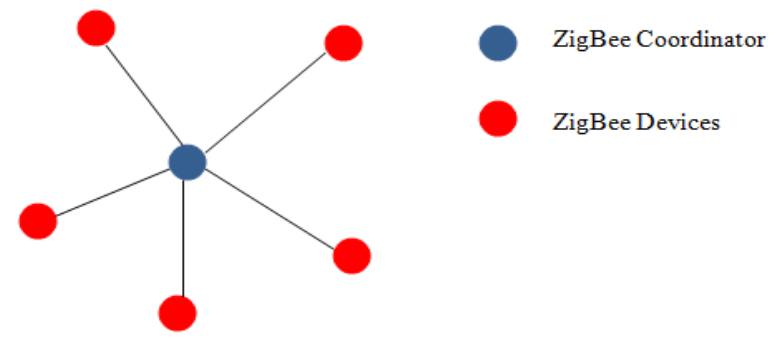

Fig 1: Star Topology

\subsection{Mesh Topology}

Figure 2 shows the mesh topology, all the nodes are connected to one another. This topology is more robust as the failure of one node does not disrupt the whole network [13].

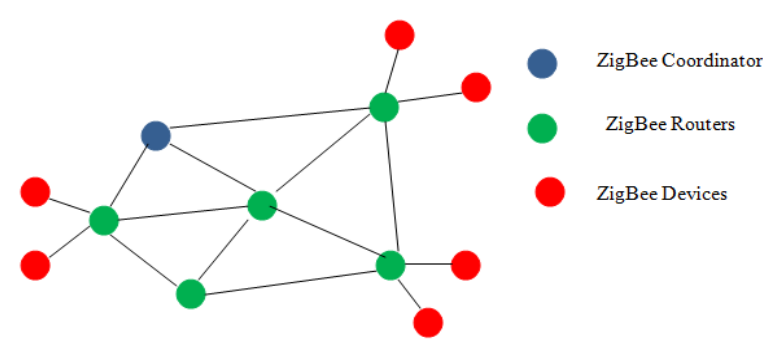

Fig 2: Mesh topology

\subsection{Tree Topology}

Figure 3 shows the tree network, the root of the network is coordinator and the end devices are connected to the router. In this topology the message is transmitted in hierarchical manner [13].

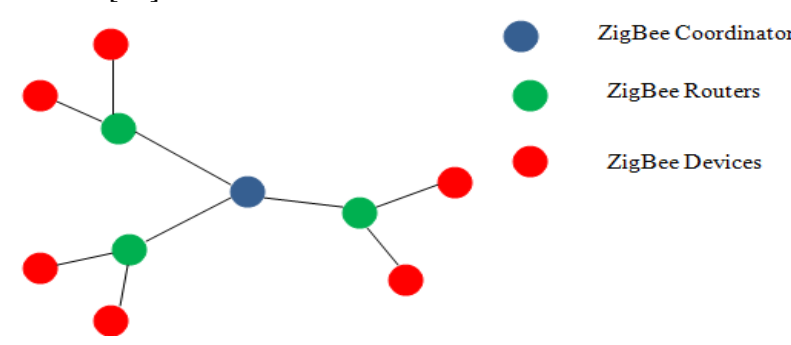

Fig 3: Tree topology 


\section{SIMULATION RESULT}

The main purpose of simulation model is to predict the performance of the real system. For analyzing the behavior of topologies in different bands the OPNET modeler is used. This modeler supports three types of topologies: star, mesh and tree $[14,15]$.

\subsection{Simulation Scenarios}

Figure 4 shows three different topological scenarios: Star, Tree and Mesh. Star topology as shown in Figure 4(a) contains one coordinator and fifteen end devices. Coordinator is a fully functional device which manages their local networks and communicates with end devices. In order to communicate, each end device has to communicate to the coordinator first and then the coordinator communicates to the destination end device i.e. no two end devices can directly communicate but only through the coordinator. Figure 4(b) shows tree topology that contains one coordinator, three routers and twelve end devices. To communicate, each end device communicates with their respective routers first and then router communicates to the coordinator and then coordinator to the destination end device. Figure 4(c) contains one coordinator, three routers and twelve end devices forming a mesh topology.

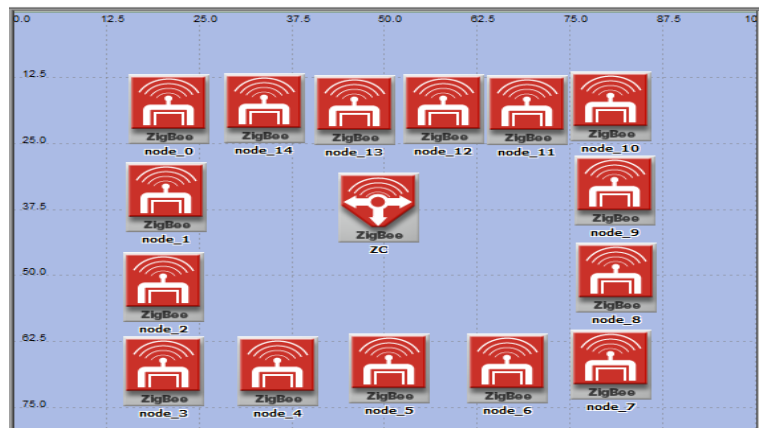

Fig 4(a): Star Topology

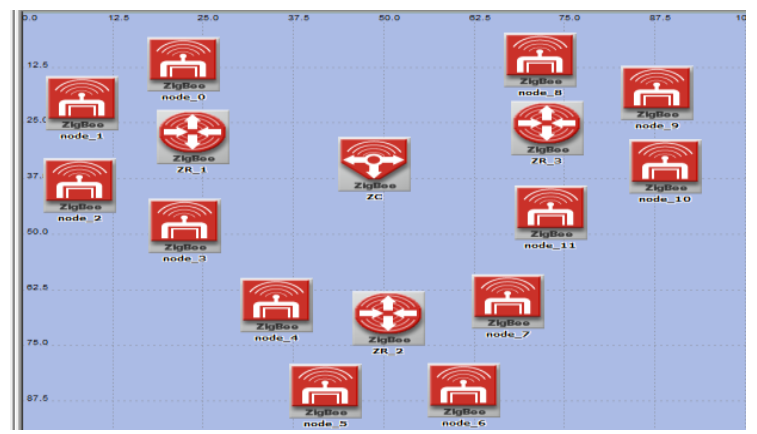

Fig 4(b): Tree Topology

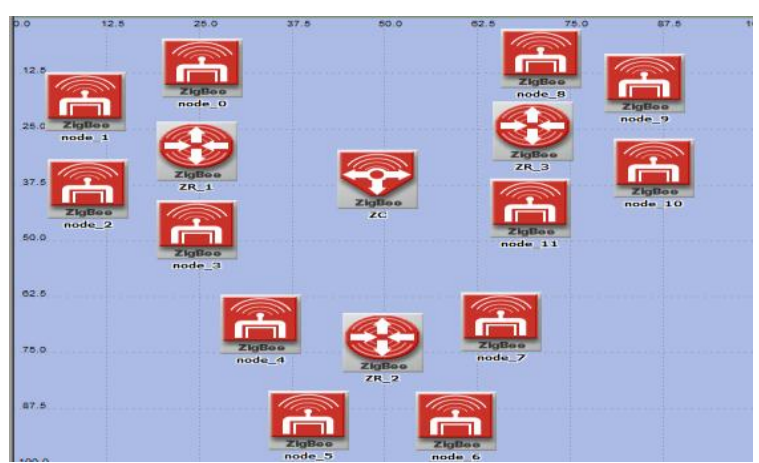

Fig 4(c): Mesh topology
Each scenario has following parametric values for different bands i.e. $2450 \mathrm{MHz}, 915 \mathrm{MHz}$ and $868 \mathrm{MHz}$ as shown in Table 1:

Table 1 Parameters used in scenario

\begin{tabular}{|l|l|l|l|}
\hline Parameters & Star & Tree & Mesh \\
\hline $\begin{array}{l}\text { Number of } \\
\text { routers }\end{array}$ & 0 & 3 & 3 \\
\hline $\begin{array}{l}\text { Minimum } \\
\text { Backoff } \\
\text { Exponent }\end{array}$ & 3 & 3 & 3 \\
\hline $\begin{array}{l}\text { Maxmium } \\
\text { Number of } \\
\text { Backoffs }\end{array}$ & 4 & 4 & 4 \\
\hline $\begin{array}{l}\text { ACK } \\
\text { mechanism }\end{array}$ & Enabled & Enabled & Enabled \\
\hline $\begin{array}{l}\text { Packet interval } \\
\text { time }\end{array}$ & Constant(1.0) & $\begin{array}{l}\text { Constant } \\
(1.0)\end{array}$ & $\begin{array}{l}\text { Constant } \\
(1.0)\end{array}$ \\
\hline
\end{tabular}

\subsection{Simulation Results}

\subsubsection{Delay}

The delay of a network determines the time taken by the data to travel across the network from one node to another node. The data rate of the link is the main cause of the transmission delay.

Figure 5(a), 5(b) and 5(c) shows the delay for the star, tree and mesh topologies in $2450 \mathrm{MHz}, 915 \mathrm{MHz}$ and $868 \mathrm{MHz}$ bands respectively. It was observed that in $2450 \mathrm{MHz}$ the delay was minimum in case of tree topology because it communicates with four fully functional devices called coordinators and routers which were more efficient as compared to the end devices while star topology was having only one fully functional device but in case of $915 \mathrm{MHz}$ and $868 \mathrm{MHz}$ bands the mesh topology delay was minimum. The lower bands experience a higher delay due to the low data rate in case of star topology.

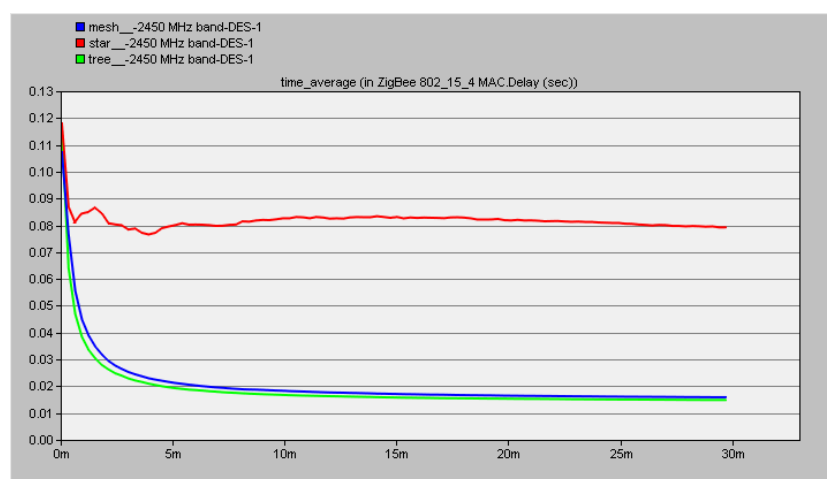

Fig 5(a): 2450 MHz band

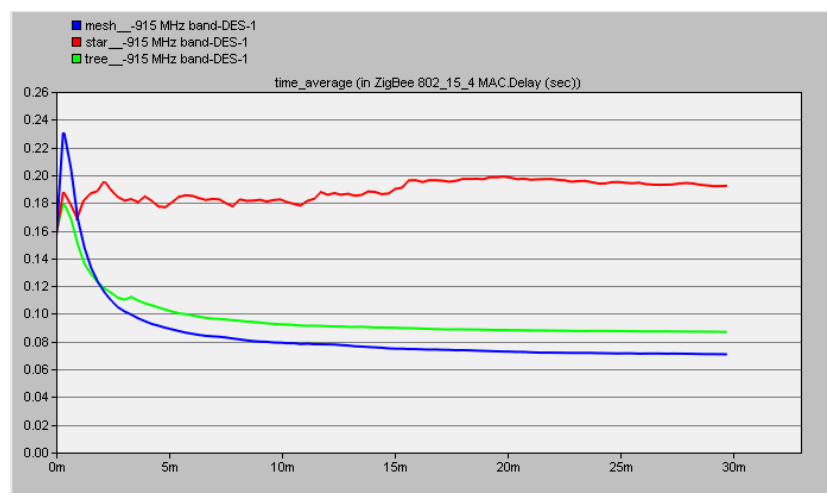

Fig 5(b): 915MHz band 


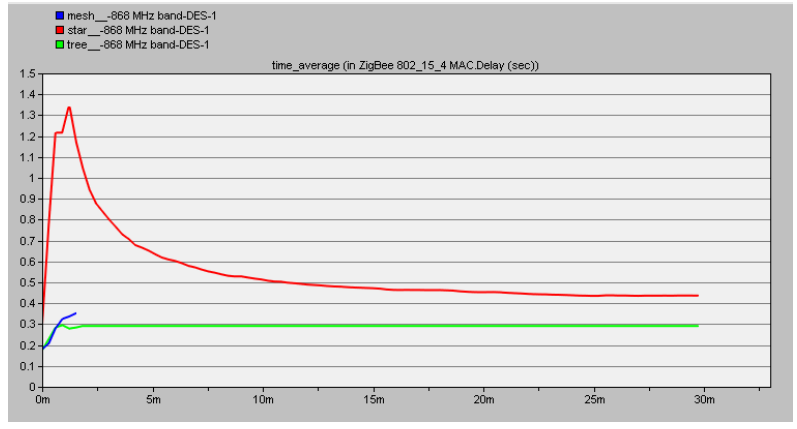

Fig 5(c): 868MHz band

\subsubsection{Load}

The total load (in bits/sec) submitted to 802.15.4 MAC is represented by all higher layers in all WPAN nodes of the network.

Figure 6(a), 6(b) and 6(c) shows the load for the star, tree and mesh topologies in $2450 \mathrm{MHz}, 915 \mathrm{MHz}$ and $868 \mathrm{MHz}$ bands respectively. It was observed that maximum load was in case of tree topology in both the bands i.e. $2450 \mathrm{MHz}$ and $915 \mathrm{MHz}$. It shows that the traffic received was maximum in tree topology because tree topology uses four coordinators and routers for communication and they were responsible for traffic generation and routing which were more efficient as compared to the end devices as compared to star topology which was having only one coordinator and fifteen end devices. In tree topology lower packet loss leads to the maximum data traffic due to the lesser collisions.

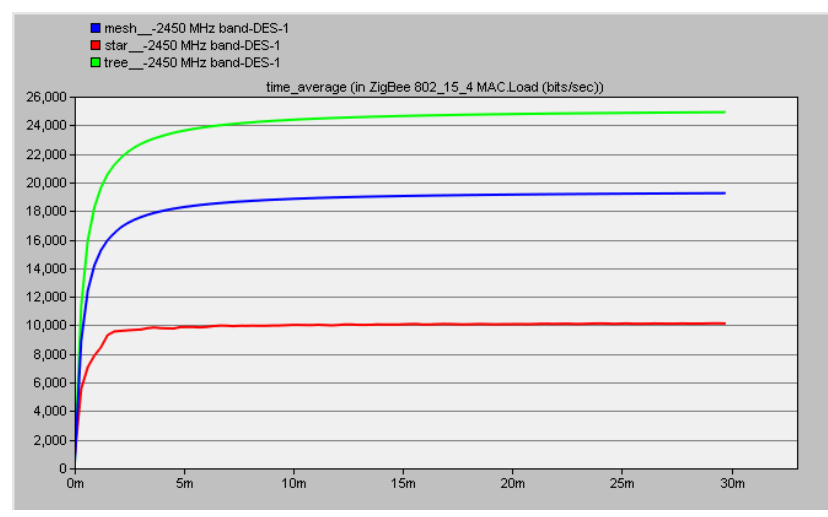

Fig 6(a): $2450 \mathrm{MHz}$ band

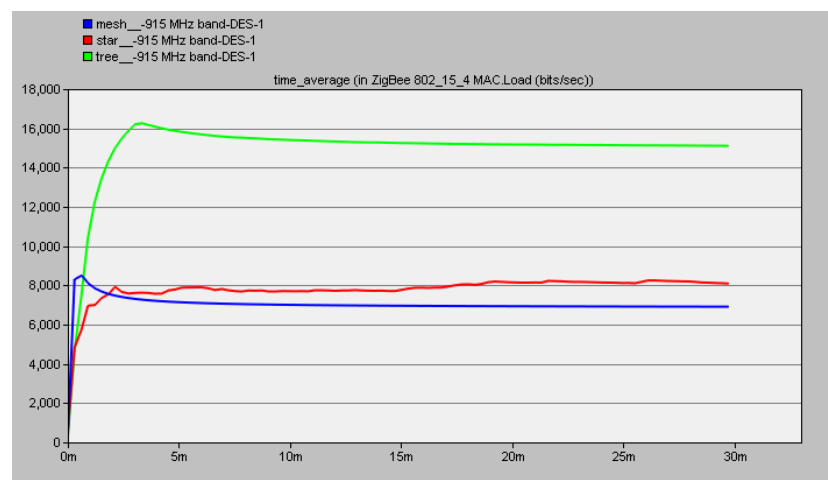

Fig 6(b): 915 MHz band

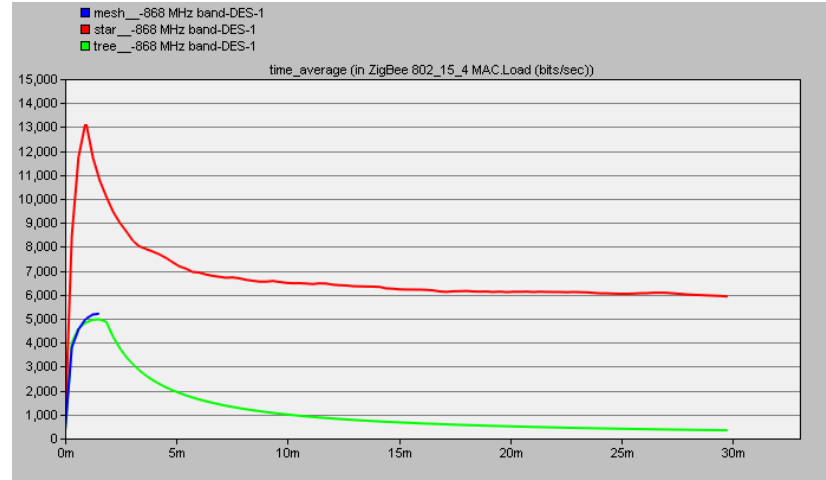

Fig 6(c): $868 \mathrm{MHz}$ band

\subsubsection{Throughput}

Throughput defines the ratio of the total amount of data that is received by a receiver from a sender to a time it takes for receiver to get the last packet. It is the average number of bits or packets successfully received or transmitted by the receiver or transmitter channel per second.

Figure 7(a), 7(b) and 7(c) shows the throughput for the star, tree and mesh topologies in $2450 \mathrm{MHz}, 915 \mathrm{MHz}$ and $868 \mathrm{MHz}$ bands respectively. It was observed that throughput was maximum in case of tree topology because it communicates with four fully functional devices called coordinator and routers which were more efficient as compared to the end devices while star topology was having only one fully functional device. This fully functional device acts as routers, repeaters, amplifiers or regenerators. In tree topology the total load of the network was divided among the routers and the coordinator due to which lesser collisions and lesser packet drops takes place as a result the throughput was maximum in case of tree topology. In mesh topology, all nodes communicate with each other, therefore the communication between ZigBee end devices were not efficient than communication between end devices to coordinator or routers. Hence in mesh topology the throughput was less than tree topology. It was also observed that throughput was minimum in case of star topology because it contains only one coordinator and fifteen end devices.

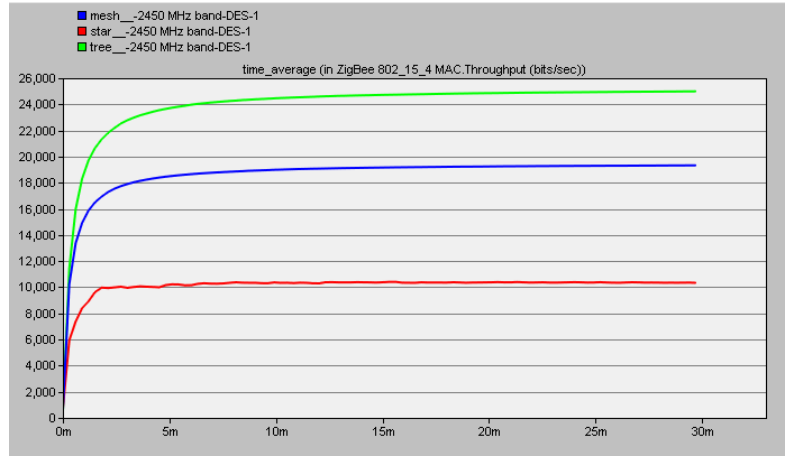

Fig 7(a): $2450 \mathrm{MHz}$ band 


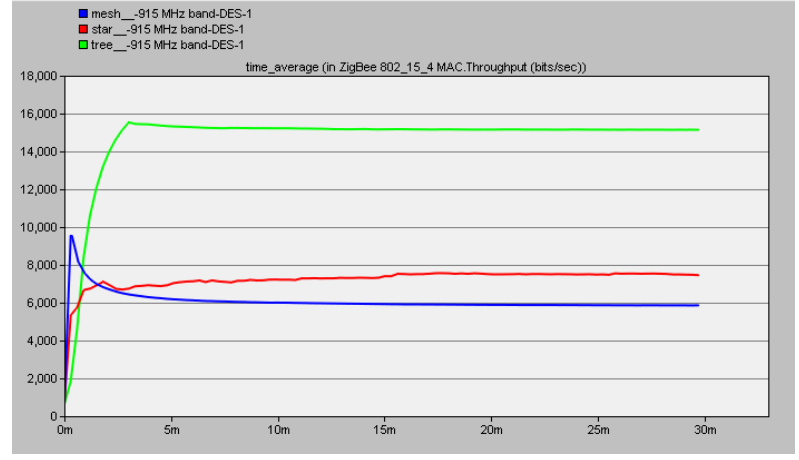

Fig 7(b): 915MHz band

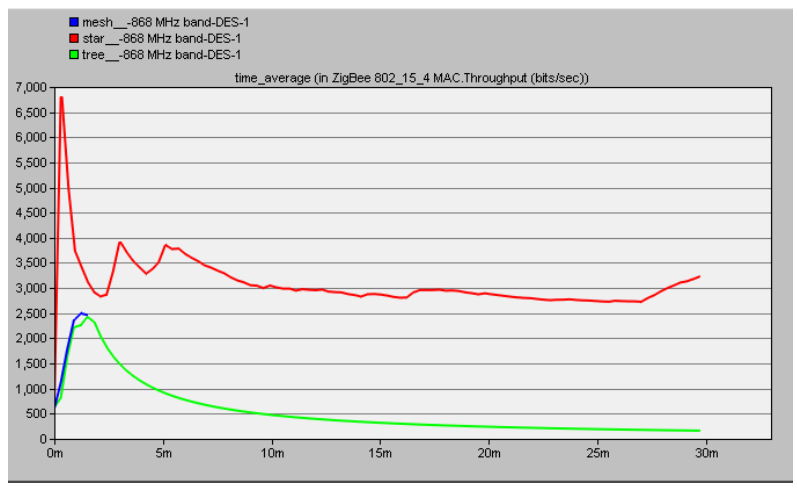

Fig 7(c): 868MHz band

\section{CONCLUSION}

This paper presents the behavior of topologies: star, tree and mesh in three different bands i.e. $2450 \mathrm{MHz}, 915 \mathrm{MHz}$ and $868 \mathrm{MHz}$ called ISM bands. The result shows that tree topology has minimum delay, maximum load and maximum throughput in $2450 \mathrm{MHz}$ band. In case of $915 \mathrm{MHz}$ band the mesh topology has minimum delay but tree topology has maximum load and throughput. In case of $868 \mathrm{MHz}$ mesh topology has minimum delay but star topology has maximum load and throughput. From the results and discussions it is concluded that tree topology is more efficient and best suited for the ZigBee wireless sensors networks as $2.4 \mathrm{GHz}$ band is more famous and it is open in most of the countries worldwide.

Our future work will be associated with the study of energyefficiency and reliability of all these topologies separately, i.e. emphasis will be placed on developing protocols that would continue the battery life, as well as access to the source code of the network and the application layers.

\section{REFERENCES}

[1] Z.Alliance, ZigBee specification v 1.0,2008.<http:// www.Zigbee.org >>.

[2] IEEE 802.15.4: Wireless Medium Access Control (MAC) and Physical Layer (PHY) Specifications for Low- Rate Wireless Personal Area Networks (LRWPANs) (2003), 3 Park Avenue, New York, USA: IEEE.
[3] Shurui Fan, Jingbo Li, Jie Li, Hexu Sun, "Performance Analysis of Wireless Network Measurement Control System using Matlab/Simulink" International Journal of Intelligent Engineering and Systems, Vol 3, No. 2, 2010.

[4] Benoît Latré, Pieter De Mil, Ingrid Moerman, Bart Dhoedt and Piet Demeester, Niek Van Dierdonck, "Throughput and Delay Analysis of Unslotted IEEE 802.15.4", Journal of Networks, Vol 1, No. 1, pp. 20-28, May 2006.

[5] Parneek kamboj, Rajan Vohra, Ravinder Singh Sawhney, "Wi-fi Performance Metrices for different Apllications using OPNET", CiiT International Journal of wireless communication, Aug 2012.

[6] N.Vlajic, D. Stevanovic, G.Spanogiannopoulos, "Strategies Performance of IEEE 802.15.4/ZigBee WSNs with Path-Constrained Mobile Sink(s)", Computer Communications, Vol 34, No. 6, pp.743-757, May 2011.

[7] Mumtaz M. Ali Al-Mukhtar and Teeb Hussein Hadi, “A Monitoring System Using Wireless Sensor Network", Journal of Al-Nahrain University, Vol 17, No. 2, pp. 219-226, June, 2014.

[8] Leung, S., Gomez, W. and Kim, J. J. "ZigBee Mesh Network Simulation using OPNET and Study of Routing Selection", spring 2009.

[9] Kinney, P. "ZigBee Technology: Wireless Control that Simply works", Communication Design Conference, 2 October 2003.

[10] Hoi Yan Tung, Kim Fung Tsang, Hoi Ching Tung, Veseline Rahocevic, Kwok Tai Chui, "A WiFi-ZigBee Building Area Network Design of High Traffics AMI for Smart Grid", Smart Grid and Renewable Energy, pp.32433, September 2012.

[11] Andrew Wheeler, Ember Corporation, "Commercial Applications of Wireless Sensor Networks Using ZigBee", Communications Magazine, Vol 45, pp. 70-77, April 2007.

[12] Ziyad K. Farej, Ali M. Abdul-Hameed, "Performance Evaluation for Large Scale Star Topology IEEE 802.15.4 Based WSN", JJEE, Vol 1, No. 1, pp. 45-54.

[13] Boris Mihajlov, Mitko Bogdanoski, "Overview and Analysis of the Performances of ZigBee- based Wireless Sensor Networks", International Journal of Computer Applications, Vol 29, No.12, pp. 28-35, Sept 2011.

[14] Rajan Vohra, R.S.Sawhney, Sunandika Mann, "Statistics Comparison in Wireless Network Envireonment for Balanced and Unbalanced Network", International Journal of Computer Applications, pp. 35-38.

[15] Rajan Vohra, R.S.Sawhney and Shikha, -WLAN Metrics Enhancement Using Buffer Size Optimization\| published in -International Journal of P2P Network Trends and Technology, Volume1, Issue2- 2011, pp. 2225. 